\title{
Charakteristische Kenngrössen schweizerischer Speicherseen
}

\section{ZUSAMMENFASSUNG}

Bei verschiedenen wasserwirtschaftlichen Problemen interessiert, wie der Inhalt eines Speichersees von dessen Stauhöhe abhängt. Es zeigt sich, dass dieser Zusammenhang, welcher natürlich wesentlich durch die Form des betreffenden Staubeckens bestimmt wird, im allgemeinen durch eine einfache mathematische Beziehung gut angenähert werden kann. Aufgrund der entsprechenden Näherungsformel gibt es zu jedem Speichersee zwei Parameter, die seine Beckenform weitgehend charakterisieren. Im vorliegenden Bericht sind für rund 40 schweizerische Speicherseen die ermittelten Parameterwerte festgehalten, sowie die sogenannten Speicherinhaltslinien aufgezeichnet. Es ist bemerkenswert, dass diese Linien, auf doppelt-logarithmischem Papier dargestellt, bei allen untersuchten Speicherseen nahezu die Gestalt von Geraden aufweisen.

\section{EINLE ITUNG}

Durch den intensiven Ausbau der Wasserkräfte vor allem in den Jahren zwischen 1945 und 1970 - sind in der Schweiz zahlreiche Stauseen (Speicherseen) entstanden. Das Eidg. Amt für Wasserwirtschaft fuihrt in seiner neuesten Statistik, Stand 1. Januar 1977, 51 schweizerische Speicherseen (im folgenden auch kurz "Speicher" genannt) mit je einem Gesamtinhalt von mehr als 10 Mio m. auf (1). In dieser Aufzählung sind Naturseen mit Absenkung bzw. Stauerhöhung inbegriffen. Von wenigen Ausnahmen abgesehen, liegen diese Speicherseen ausschliesslich in den Alpen.

Jede Standortwahl von künstlich angelegten Speichern bzw. von Talsperren erfordert umfassende Untersuchungen und Vorabklärungen. Verschiedene Kriterien sind dabei zu berücksichtigen: hydrologische, geologische und geomorphologische Voraussetzungen, Energiebilanz, Natur.und Heimatschutz, ökologische Gesichtspunkte u.a.m. Wenn alternative Standorte von Talsperren gegeneinander abzuwägen sind, müssen unter anderem auch die Volumina der entsprechenden alternativen Staubecken miteinander verglichen werden. Dabei interessieren nicht nur der Gesamtinhalt der Becken, sondern auch die Inhalte, welche variablen Seeständen entsprechen. In diesem Zusammenhang, aber auch bei anderen wasserwirtschaftlichen Problemen, besteht das Be- dürnis nach einer einfachen Methode, die es ermöglicht, den Speicherinhalt bei verschiedenen Stauhöhen - wenigstens näherungsweise - zu ermitteln. Im folgenden wird gezeigt, dass sich zu jedem Speichersee zwei Parameter finden lassen, die die Form des betreffenden Staubeckens weitgehend charakterisieren. Mittels dieser $\mathrm{Pa}-$ rameter kann der Speicherinhalt mit guter Nährung bestimmt werden.

Unter der Stauhöhe $\mathrm{H}$ eines Speichersees wird hier stets die Höhendifferenz zwischen dem Wasserspiegel und dem tiefsten Seegrund verstanden. Der Speicherinhalt, welcher der Stauhöhe $H$ entspricht, wird mit $V$ bzw. $V(H)$ bezeichnet. $F$ bzw. $F(H)$ gibt die Stauspiegelfläche, d.h. das Flächenmass des der Stauhöhe $H$ entsprechenden Wasserspiegels an.

\section{Speicherkennlinien}

Uịe Abhängigkeit des Speicherinhaltes $V$ von der Stauhöhe $H$ wird - meistens in graphischer Form durch die sogenannte Speicherinhaltslinie wiedergegeben. Diese Linie charakterisiert weitgehend die Form des Staubeckens. So wie die einzelnen Stauseen zum Teil recht unterschiedliche Beckenformen aufweisen, zeigen sich auch deutliche Unterschiede im Verlauf der betreffenden Speicherinhaltslinien.

Eine weitere Speicherkennlinie ist die sogenannte Linie der Stauspiegelfläche. Sie zeigt, wie sich die Stauspiegelfläche F bei variierender Stauhöhe $H$ ändert. Gewöhnlich wird die Stauspiegelfläche für diskrete Werte der Stauhöhe aus topographischen Karten planimetrisch bestimmt. Aus der ermittelten Abhängigkeit $F=F(H)$ ergibt sich dann die Speicherinhaltslinie durch Integration:

$$
V(H)=\int_{0}^{H} F(h) d h
$$$$
-1-
$$

Das Bild I zeigt als Beispiel die Speicherinhaltslinie und die Linie der Stauspiegelfläche des Göscheneralpsees.

Dr. Anton Kuihne, Versuchsanstalt für Wasserbau, Hydrologie und Glaziologie an der ETH, 8092 Zürich. 


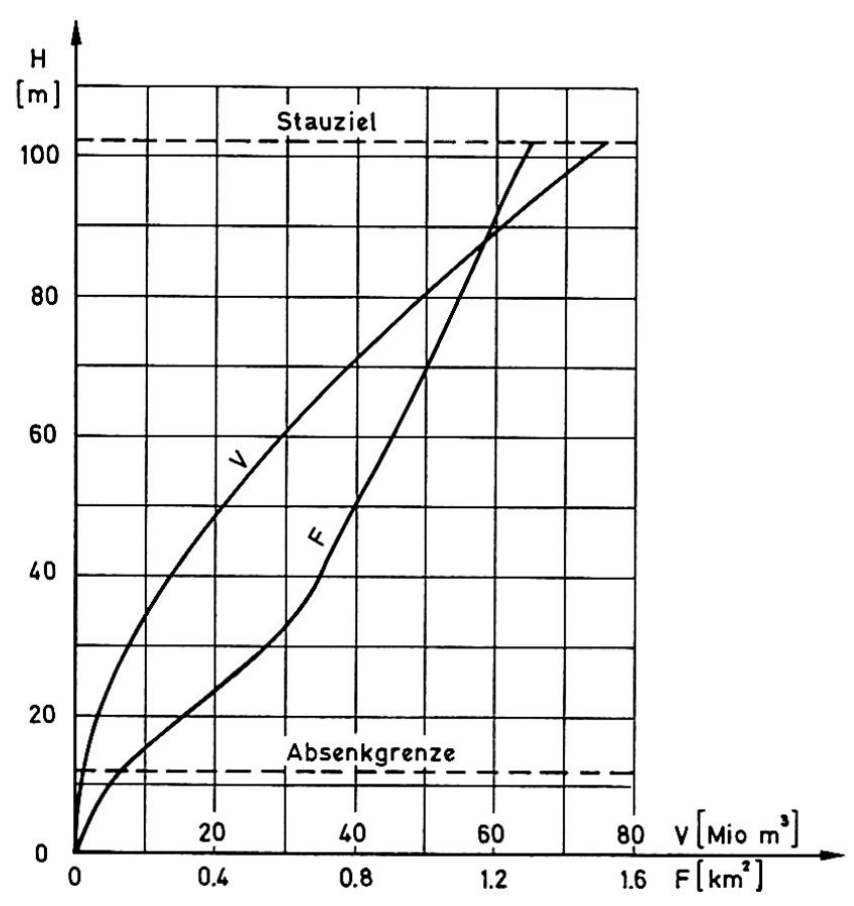

Bi.ld 1: Speicherinhaltslinie und Linie der Stauspiegelfläche des Göscheneralpsees

2. Näherungsformeln für Speicherinhalt und Stauspiegelfläche

Die Abhängigkeit des Speicherinhaltes von der Stauhöhe gehorcht im allgemeinen nicht genau einer einfachen, formelmässig ausdrückbaren Beziehung. Dies erklärt sich aus den Unregelmässigkeiten in der Geländeform des eingestauten Talabschnittes. Aus wasserwirtschaftlicher sicht wäre natürlich eine einfache, analytische Beziehung $V=V(H)$ erwünscht, welche den wirklichen Zusammenhang zwischen $V$ und $H$ mit hinreichenderGenauigkeit beschreiben würde. Dies könnte verschiedene Berechnungen sowohl in der Planungsphase, als auch beim Betrieb von Speichern erleichtern. Daher liegt der Versuch nahe, die wirklichen Speicherinhaltslinien durch geeignete einfache, analytische Funktionen anzunähern. Verschiedene Autoren haben nachgewiesen, dass sich der Speicherinhalt im allgemeinen gut durch den Ansatz

$$
V(H)=a H^{b}
$$

approximieren lässt $(2,3,4)$. Dabei sind a und b zwei konstante Beiwerte, welche die Form des Staubeckens weitgehend charakterisieren. Sie sind für jeden Speicher geeignet zu wählen, so dass die dem Potenzgesetz -2- entsprechende Kurve möglichst gut mit der wirklichen (gemessenen bzw. ermittelten) Speicherinhaltslinie übereinstimmt. Wie a und b zweckmässig bestimmt werden, wird später kurz beschrieben. Die Speicherinhaltslinie und die Linie der Stauspiegelfläche sind durch die Beziehung -1-miteinander verknüpft. Entsprechend dem Ansatz -2wird daher die Linie der Stauspiegelfläche eben- falls durch ein Potenzgeset.z approximiert, nämlich

$$
F(H)=a b H^{b-1}
$$

\section{Bedeutung der Beiwerte $a$ und $b$}

\subsection{Beiwert b}

Der wesentliche Informationsgehalt uber die Form eines Speicherbeckens liegt im Exponenten b der approximierenden Funktion -2-. Am Beispiel einiger stark idealisierter Beckenformen kann qualitativ gezeigt werden, wie die eingestaute Talform durch den Beiwert b -eine dimensionslose Grösse-wiedergegeben wird. Das Bild 2 zeigt je 4 verschiedene Längs- und Querschnittsformen. Durch den Stauspiegel und die 4 gezeigten Längsschnitte bzw. Querschnitte wird je eine Fläche $F_{p}$ bzw. $F_{g}$ definiert, für die die folgende Beziehung gilt:

$$
\begin{aligned}
& F_{p}=c H^{p} \text {, bzw. } \\
& F_{q}=c H^{q} .
\end{aligned}
$$

$c, p$ und $q$ sind konstante Beiwerte. Kombiniert kennzeichnen die 4 Längs- und Querschnitte insgesamt 16 idealisierte Beckenformen. Deren Speicherinhalt als Funktion der Stauhöhe $H$ sei wiederum als $V=a H^{b}$ angesetzt. Der Exponent $b$ setzt sich aus $p$ und $q$ näherungsweise wie folgt zusarnmen:

$$
b=p+q-1
$$

\begin{tabular}{|c|c|c|c|c|c|}
\hline & & \multicolumn{4}{|c|}{ Längsschnitt } \\
\hline & & I & I I & I I I & IV \\
\hline \multirow{4}{*}{ 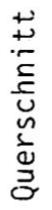 } & 1 & 1 & 1.5 & 2 & 2.5 \\
\hline & 2 & 1.5 & $2^{\star}$ & 2.5 & $3 *$ \\
\hline & 3 & 2 & 2.5 & 3 & 3.5 \\
\hline & 4 & 2.5 & $3^{\star}$ & 3.5 & $4^{\star}$ \\
\hline
\end{tabular}

Für jene idealisierten Beckenformen, welche in Tabelle 1 nicht mit * markiert sind, gilt diese Beziehung sogar exakt.

Tab. 1: Abhängigkeit des Exponenten $b$ von den Beckenformen aus Bild 2

Natürlich werden kaum reale Stauseen anzutreffen sein, die genau einer der idealisierten Beckenformen gemäss Bild 2 entsprechen. Gleichwohl ergeben sich aus jener Darstellung bzw. aus der Tabelle 1 einige interessante Hinweise über den Zusammenhang zwischen Beckenform und Beiwert b. Speicherseen mit $b<1$, also Becken, die sich nach oben verengen, kommen praktisch nicht vor. $b=1$ würde bedeuten, dass das Flächenmass des Wasserspiegels für alle Stauhöhen gleich bliebe und der Speicherinhalt daher proportional zur Stauhöhe wäre. Die Speicher in der Praxis haben Bei- 


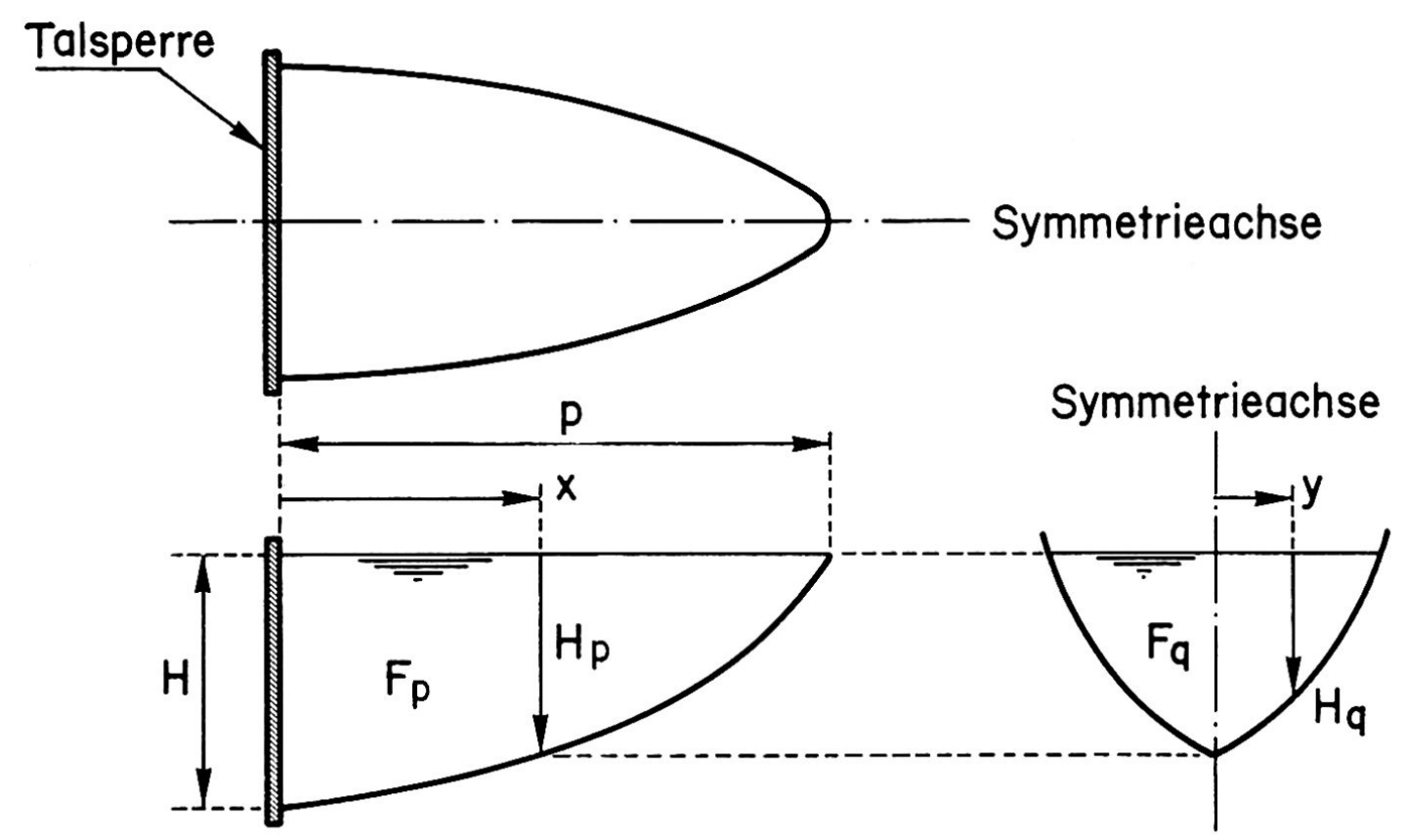

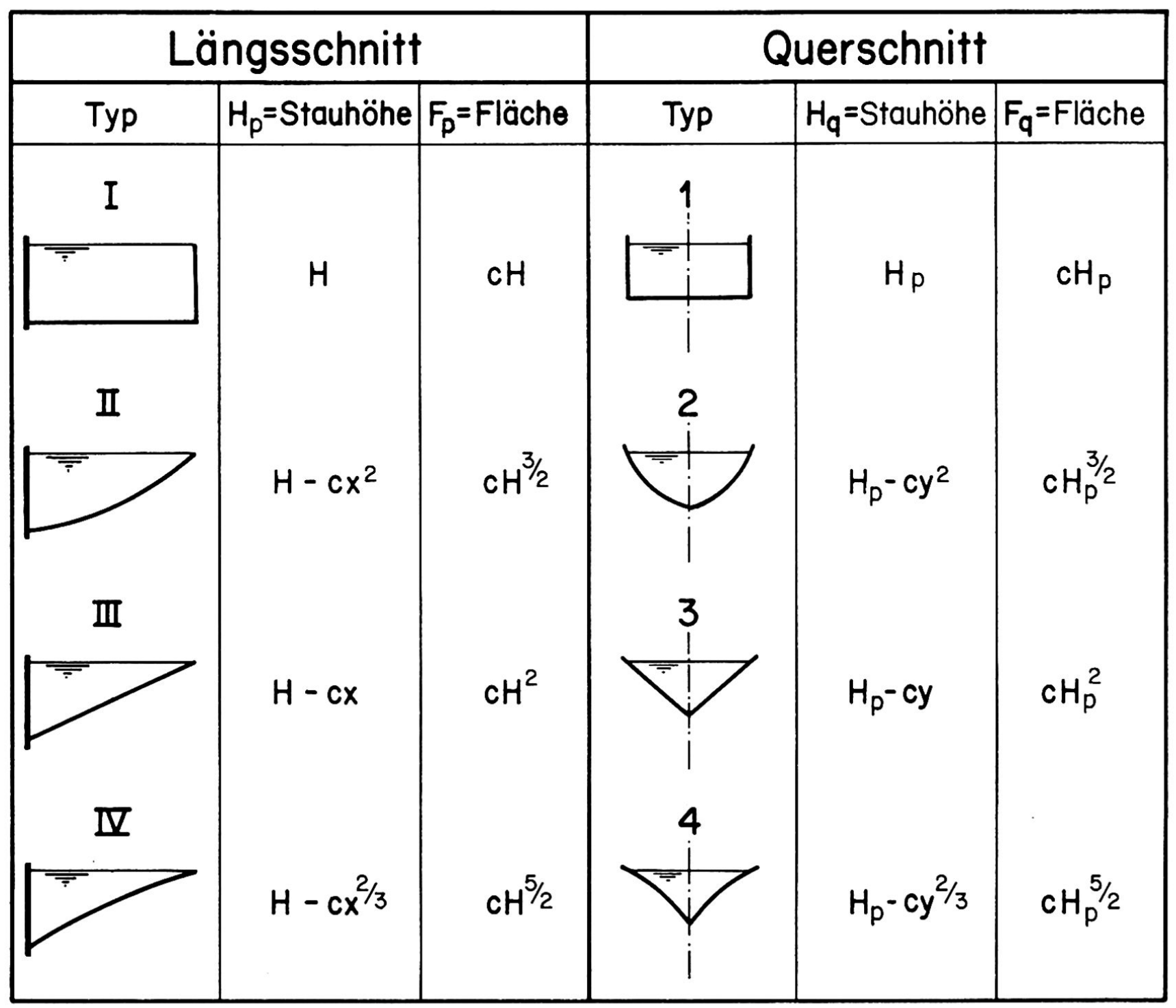

Bilf 2: Idealisierte Talformen im Längs- und im Querschnitt $(c=$ konst. Faktor) 
werte $b>1$. Die Ueberprüfung des Ansatzes $V=$ a $\mathrm{Hb}$ an 61 schweizerischen Speicherseen ergab ausschliesslich Werte innerhalb des Intervalles $1.3<b<4.0$.

Der Beiwert $b$ charakterisiert weitgehend die Beckenform eines Speichers. Bei Becken mit relativ kleinen b-Werten nimmt die Stauspiegelfläche mit wachsender Stauhöhe nur langsam zu. Grosse Werte von b, etwa $b>3$, kennzeichnen Staubecken, die sich nach oben sehr rasch erweitern. b bzw. (b-1) ist ein Mass dafür, wie stark der Speicherinhalt bzw. die Stauspiegelfläche mit zunehmender Stauhöhe anwächst. Hingegen kann aufgrund des Beiwertes b nicht im einzelnen auf die Längs- und Querschnittsform des betreffenden Speichers und auch nicht auf die geometrische Form der Stauspiegelfläche geschlossen werden. So zeigt die Tabelle 1 , dass $z$.B. die vier verschiedenen Kombinationen I 4, II 3, III 2 und IV 1 je auf eine idealisierte Beckenform mit $b=2.5$ führen.

Der Beiwert b gibt auch einen Hinweis in Bezug auf den Abstand zwischen den Höhenkurven auf topographischen Karten. Ein kleiner b-Wert ist typisch für einen Stausee mit einem relativ flachen Seegrund und teilweise recht steilen "Wänden". Dementsprechend liegen in Speicherseen mit kleinen b-Werten die Höhenkurven mit den niedrigsten Koten relativ weit auseinander; im Bereich von grossen Stauhöhen, also in der Nähe des Stauzieles, werden hingegen die Abstände zwischen aufeinanderfolgenden Höhenkurven kleiner (Beispiele: Lai da Marmorera, Mattmarksee). Speicherseen mit grossen b-Werten zeichnen sich demgegenüber durch enger beisammenliegende Höhenkurven im untersten Bereich des Staubeckens aus (Beispiele: Lac de l'Hongrin, Lac de la Gruyère).

\subsection{Beiwert a}

Wird die Stauhöhe $H$ in Metern gemessen, so nimmt der Beiwert a die Einheit $m^{3-b} a n$. Wegen -2und -3-gibt dann der numerische Wert von a theoretisch den Beckeninhalt (in $\mathrm{m}^{3}$ ) bei $\mathrm{H}=1 \mathrm{~m}$ an, während der numerische Wert des Produktes a b die Spiegelfläche (in $\mathrm{m}^{2}$ ) bei $\mathrm{H}=1 \mathrm{~m}$ ausdruickt. Diese Werte haben allerdings eher symbolische als praktische Bedeutung, weil der Ansatz $V=a H^{b}$ gerade im Bereich von sehr geringen Stauhöhen $H$ den wirklichen Speicherinhalt relativ ungenau wiedergibt.

Der Beiwert a kann von Becken zu Becken stark schwanken. Die entsprechenden Werte der schweizerischen Speicherseen liegen im Bereich $1<a<10^{6}$. Der Wert von a muss im ubrigen immer im Zusammenhang mit jenem von b gesehen werden. Grossen (bzw. kleinen) b-Werten entsprechen kleine (bzw. grosse) a-Werte. Bei Speichern mit gleichem Exponent b, aber unterschiedlichem Beiwert a, kann auf unterschiedliche Ausdehnung der einzelnen Staubecken geschlossen werden. Die grösseren Werte von a kennzeichnen dann jene Becken, welche bei gleicher Stauhöhe die grössere Seeoberfläche aufweisen.

\subsection{Numerische Bestimmung von $a$ und $b$}

Wie sind nun die für einen Speichersee passenden Beiwerte a und b konkret zu ermitteln? Aufgrund der beiden Ansätze -2- und -3-gilt für $b$ die Beziehung:

$$
b=\frac{F H}{V}
$$

Dies bedeutet, dass sich der Beiwert b auf einfache Weise berechnen lässt, wenn für eine bestimmte Stauhöhe der entsprechende Speicherinhalt und die Stauspiegelfläche bekannt sind. Ist $b$ bestimmt, so ergibt sich a $z u a=V / H^{b}$. Freilich sind die so ermittelten Werte für a und $b$ nur als eine erste, grobe Annäherung zu betrachten. Wenn eine gute Näherung über den ganzen Schwankungsbereich der Stauhöhe $H$ gesucht ist, sind die Beiwerte $a$ und $b$ der approximierenden Funktion $V=a H^{b}$ durch ein Ausgleichsverfahren aus der wirklichen Speicherinhaltslinie zu ermitteln. Dies führt dann auf ein sogenanntes nichtlineares Ausgleichsproblem. Letzteres kann durch ein einfaches graphisches oder, mit grösserer Genauigkeit, durch ein numerisches Verfahren gelöst werden. Beide Methoden sind in (5) näher beschrieben. Hier soll nur kurz das graphische Verfahren skizziert werden.

Auf doppelt-logarithmischem Papier aufgezeichnet, nimmt die Beziehung $V(H)=a H^{5}$ exakt die Gestalt einer Geraden an:

$$
\log (V)=\log (a)+b \log (H)
$$

Die Wertepaare $(\mathrm{V}, \mathrm{H})$ aus der vorgegebenen Speicherinhaltslinie werden daher als Punkte auf doppelt-logarithmischem Papier aufgetragen. Durch diese Punktmenge wird graphisch eine Ausgleichsgerade gelegt. Dabei ist auf eine gute Uebereinstimmung vor allem im oberen Bereich (grosse $\mathrm{H}$ - und V-Werte) zu achten. Die beiden gesuchten Beiwerte a und $b$ ergeben sich dann aus dem Achsenabschnitt bei $H=1 \mathrm{~m}(\log (H)=0)$ bzw. aus der Steigung der Ausgleichsgeraden, siehe Bild 3 .

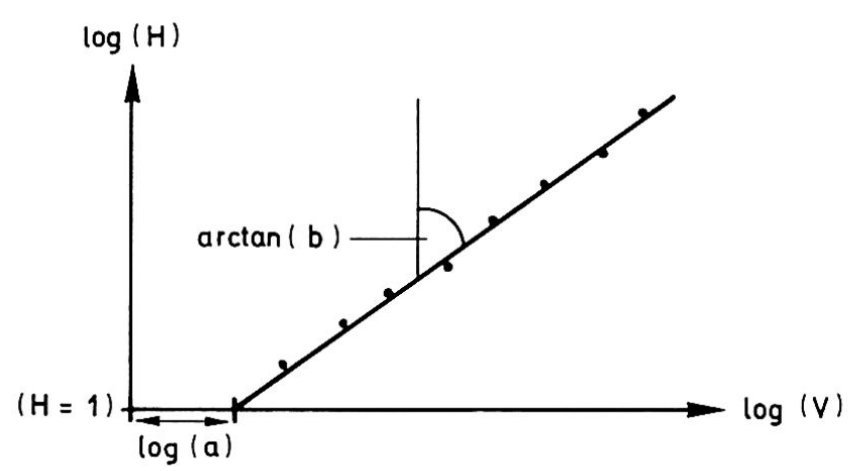

bild 3: Bestimmung der Beiwerte a und b auf doppelt-logarithmischem Papier

4. Speicherinhaltslinien schweizerischer Stauseen

Im Rahmen einer Studie wurden die Speicherinhaltslinien von 61 schweizerischen Stauseen unter- 


\begin{tabular}{|l|r|l|c|}
\hline Speicher & $\begin{array}{c}\mathrm{V}_{\max } \\
\left(\text { Mio m }^{3}\right)\end{array}$ & $\begin{array}{c}\mathrm{a} \\
\left(\mathrm{m}^{3-\mathrm{b}}\right)\end{array}$ & $\begin{array}{c}\mathrm{b} \\
(-)\end{array}$ \\
\hline Lago di Lei $\left.{ }^{*}\right)$ & 200.0 & $8.9710^{1}$ & 2.99 \\
Zervreilasee & 100.5 & $4.4810^{3}$ & 2.03 \\
Lai da Sta.Maria & 67.3 & $3.8510^{2}$ & 2.69 \\
Lai da Marmorera & 62.6 & $1.9610^{5}$ & 1.38 \\
Lai da Nalps & 45.1 & $1.3110^{2}$ & 2.66 \\
Lai da Curnera & 41.1 & $2.7810^{2}$ & 2.49 \\
Gigerwald & 35.6 & $3.1710^{1}$ & 2.84 \\
Sufnersee & 21.4 & $3.3410^{3}$ & 2.23 \\
\hline
\end{tabular}

Tab. 2: Speicherkenngrössen, Flussgebiet Rhein ( $V_{\text {max }}=$ Gesamtinhalt bis zum Stauziel)

*) Speichersee liegt auf italienischem Territorium, die Nutzung erfolgt auf schweizerischem Gebiet. sucht(5). Von diesen Seen standen Pläne des Eidg. Amtes für Wasserwirtschaft zur Verfügung, welche die Linie der Stauspiegelfläche sowie die sogenannte Nutzinhaltslinie enthalten. Letztere gibt zu bestimmten Höhenkoten HK den Inhalt zwischen HK und der Absenkgrenze des Speichers an. Wenn die Höhendifferenz $\mathrm{H}_{0}$ zwischen der Absenkgrenze und dem tiefsten Seegrund sowie das entsprechende Restvolumen $V_{0}$ bekannt ist, kann aus der Nutzinhaltslinie die Speicherinhaltslinie abgeleitet werden, jedoch nur für den Bereich oberhalb der Absenkgrenze, den sogenannten Nutzbereich. Die im folgenden festgehaltenen Resultate basieren auf den genannten Unterlagen, sowie auf einer tabellarischen Uebersicht, die vom Eidg. Amt für Wasserwirtschaft publiziert worden ist (6). In den Bildern 4 bis 7 sind - im Nutzbereich die Speicherinhaitslinien von 38 schweizerischen Stauseen, welche je ein Fassungsvermögen von mindestens $20 \mathrm{Mio} \mathrm{m}^{3}$ aufweisen, wiedergegeben. Diese Darstellungen zeigen, dass die Speicherinhaltslinien auf doppelt-logarithmischem Papier im allgemeinen nur wenig von der

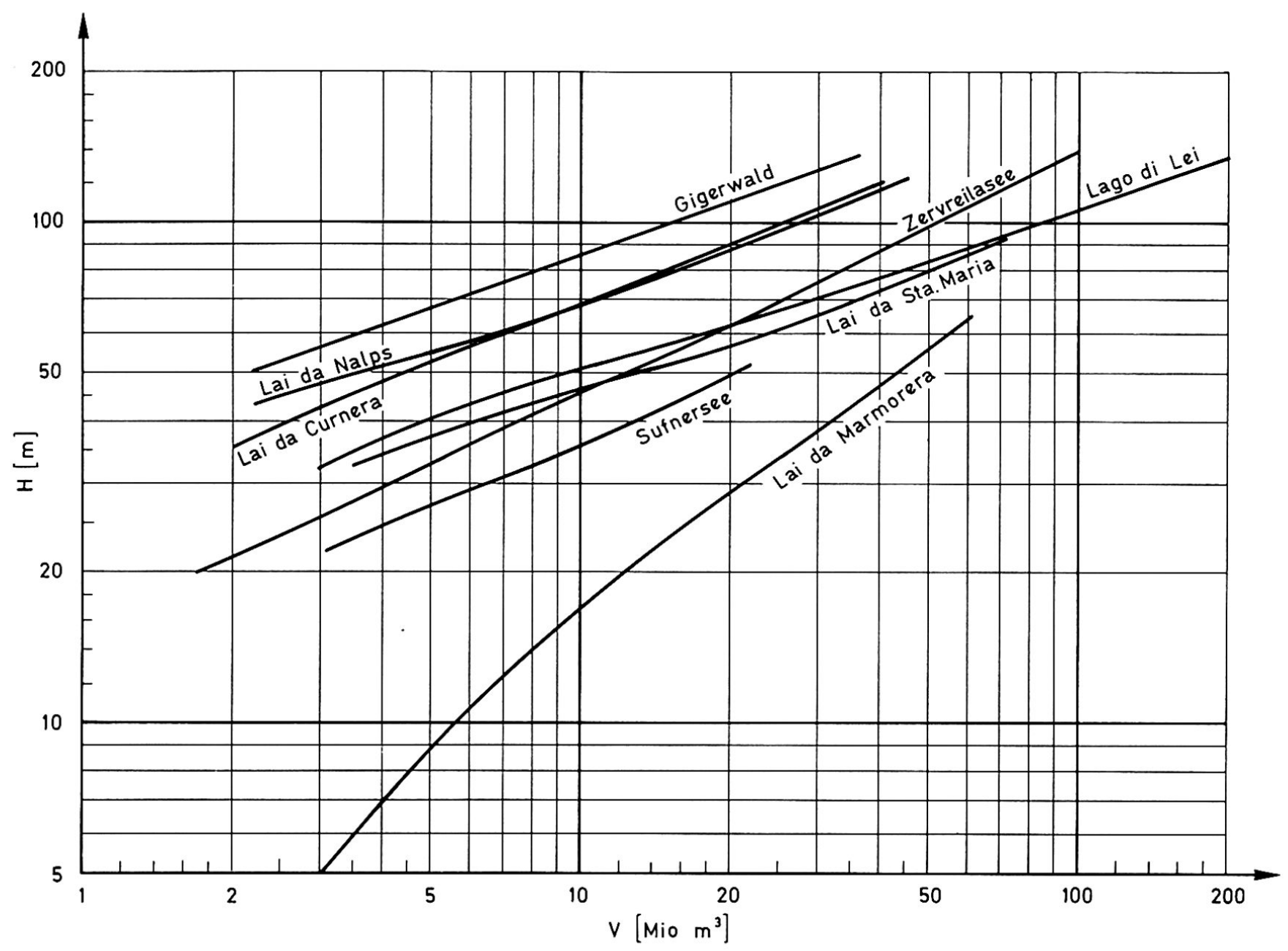

Bild 4: Speicherinhaltslinien, Flussgebiet Rhein

( $H=$ Stauhöhe, $V=$ Speicherinhalt) 


\begin{tabular}{|l|r|ll|c|}
\hline Speicher & $\begin{array}{c}\mathrm{V}_{\max } \\
\left(\text { Mio } \mathrm{m}^{3}\right)\end{array}$ & $\begin{array}{c}\mathrm{a} \\
\left(\mathrm{m}^{3-\mathrm{b}}\right)\end{array}$ & $\begin{array}{c}\mathrm{b} \\
(-)\end{array}$ \\
\hline Lac de la Gruyère & 200.0 & 4.22 & $10^{1}$ & 3.56 \\
Grimselsee & 101.0 & 1.71 & $10^{2}$ & 2.89 \\
Schiffenensee & 66.0 & 5.91 & $10^{3}$ & 2.56 \\
Oberaarsee & 60.7 & 7.07 & $10^{2}$ & 2.52 \\
Lac de l'Hongrin & 53.2 & 2.44 & $10^{1}$ & 3.13 \\
Räterischsbodensee & 26.3 & 4.37 & $10^{3}$ & 2.02 \\
Göscheneralpsee & 76.0 & 1.79 & $10^{4}$ & 1.81 \\
Lungernsee & 65.0 & 1.06 & $10^{4}$ & 2.07 \\
Lago di Lucendro & 25.6 & 2.01 & $10^{3}$ & 2.07 \\
Wägitalersee & 153.0 & 8.43 & $10^{4}$ & 1.83 \\
Sihlsee & 96.5 & 1.76 & $10^{5}$ & 2.21 \\
Limmerensee & 93.0 & 9.41 & $10^{3}$ & 1.91 \\
Klöntalersee & 56.4 & 2.70 & $10^{3}$ & 2.59 \\
\hline
\end{tabular}

Tab. 3: Speicherkenngrössen, Flussgebiet Aare, Reuss, Linth/Limmat
Gestalt einer Geraden abweichen. Da die logarithmische Transformation mit einer Massstabsverzerrung verbunden ist, dürfen Abweichungen im unteren Bereich der Speicherinhaltslinie ( $H$ klein) nicht überbewertet werden. Der Näherungsansatz $V(H)=a H^{b}$ für den Speicherinhalt ist also gerechtfertigt.

Die passenden Beiwerte a und b der schweizerischen Speicherseen wurden durch ein numerisches Ausgleichsverfahren berechnet. Diese Werte definieren jene Funktionen $V(H)=a H^{b}$, welche die wirklichen Speicherinhaltslinien nach der Methode der kleinsten Quadrate am besten approximieren. Die berechneten Beiwerte $a$ und $b$, welche den Speicherinhaltslinien in den Bildern 4 bis 7 entsprechen, sind in den Tabellen 2 bis 5 festgehalten. Für jeden Speichersee können die ermittelten Werte leicht überprüft werden (vgl. Bild 3 ). In wenigen Ausnahmefällen weist die eingestaute Talform markannte Unregelmässigkeiten auf, so dass sich eine verbesserte Approximation mit zwei verschiedenen Wertepaaren (a,b) empfehlen würde, nämlich $V=a^{\prime} H^{b}$ im unteren Bereich des Staubeckens ( $H$ klein) und $V=a^{\prime \prime} H^{b "}$ im oberen Bereich (H gross).

Bei der Interpretation der Rechenergebnisse ist zu beachten, dass die Eingabedaten mit gewissen Ungenauigkeiten behaftet waren. Diese steckten zum Teil schon in den für die Studie

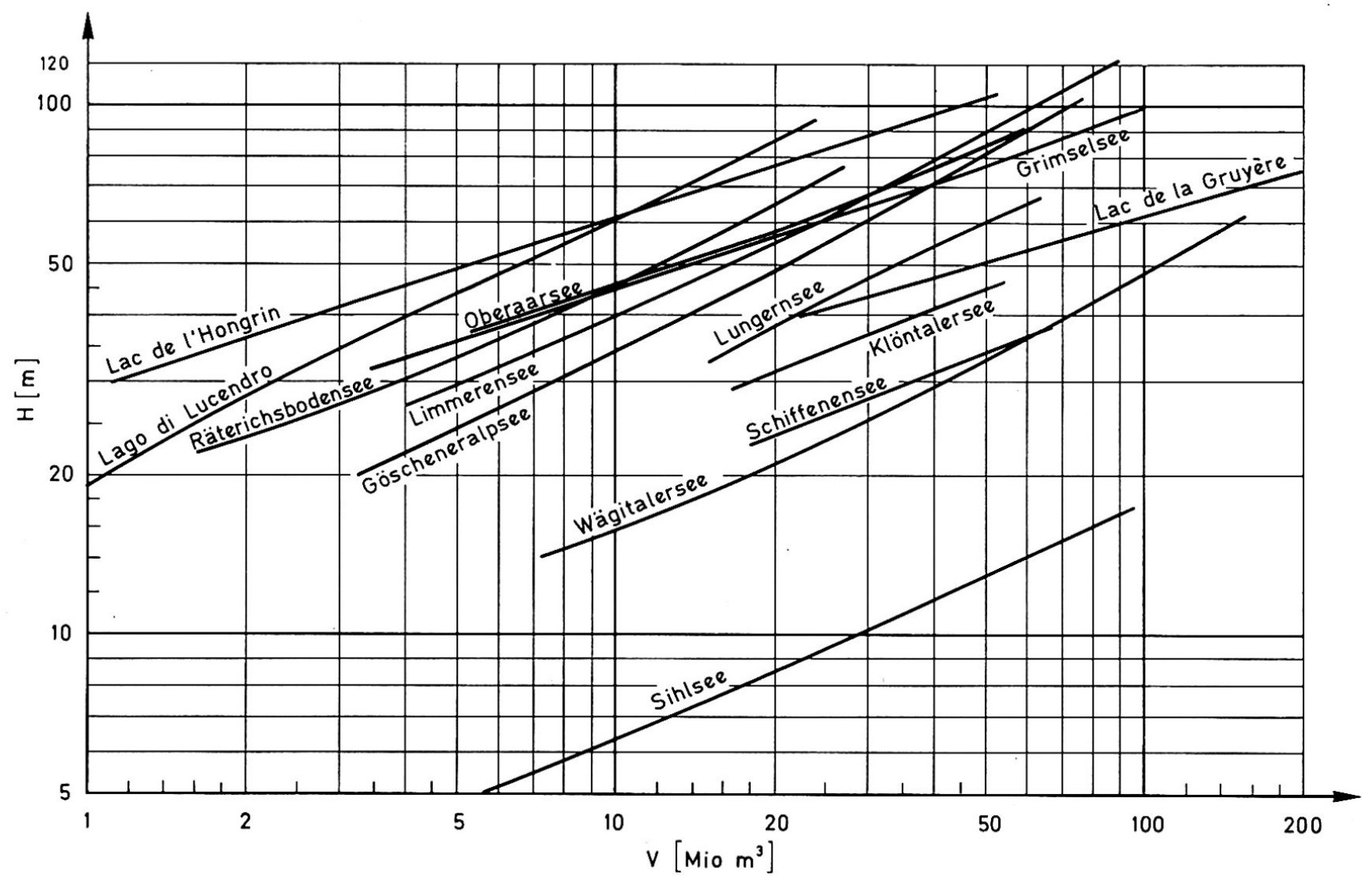

Ahh. 5: Speicherinhaltslinien, Flussgebiete Aare, Reuss, Linth/Limmat 


\begin{tabular}{|l|c|cc|c|}
\hline Speicher & $\begin{array}{c}v_{\max } \\
\left(\text { Mio } \mathrm{m}^{3}\right)\end{array}$ & $\begin{array}{c}\mathrm{a} \\
\left(\mathrm{m}^{3-\mathrm{b}}\right)\end{array}$ & $\mathrm{b}$ \\
\hline Lac des Dix & 401.0 & 2.40 & $10^{3}$ & 2.24 \\
Lac d'Emosson & 227.0 & 9.66 & $10^{2}$ & 2.44 \\
Lac de Mauvoisin & 181.5 & 5.30 & $10^{3}$ & 2.01 \\
Mattmarksee & 101.0 & 8.45 & $10^{4}$ & 1.56 \\
Lac de Moiry & 78.0 & 1.46 & $10^{4}$ & 1.83 \\
Lac de Zeuzier & 51.0 & 2.46 & $10^{3}$ & 2.07 \\
Lac de Salanfe & 40.1 & 3.42 & $10^{3}$ & 2.47 \\
Lac des Toules & 20.15 & 7.21 & $10^{2}$ & 2.38 \\
Lac de Cleuson & 20.0 & 7.23 & $10^{3}$ & 1.82 \\
\hline
\end{tabular}

Tab. 4: Speicherkenngrössen, Flussgebiet Rhône zur Verfügung stehenden Nutzinhaltslinien. Zu einem andern Teil entstanden sie beim Herauslesen von Werten (Stuitzwerten) aus diesen Linien. Ueber die Beckenform und den Beckeninhalt unterhalb der Absenkgrenze lagen für einzelne Speicher in den Unterlagen nur ungenaue Angaben vor. Es ist zu beachten, dass sich die "Anfangswerte" $H_{0}$ und $V_{0}$ im Laufe der Zeit verändern können, als Folge von Verlandungen. Dementsprechend muissten die Speicherinhaltslinien korrigiert und die Beiwerte $a$ und $b$ angepasst werden. Dies würde dann zu etwas kleineren b-Werten führen.

Bei den 61 untersuchten schweizerischen Speicherseen schwankt der Exponent b zwischen den Werten 1.38 (Lai da Marmorera) und 3.56 (Lac de la Gruyère), mit einem arithmetischen Mittelwert von 2.22. Beiläufig sei erwähnt, dass Benk (3) bei der Auswertung von insgesamt 24 Hochwasserrückhaltebecken im Schwarzwald, im Schwäbischen Wald und im Jagstgebiet ähnliche Ergebnisse erhalten hatte. Dort bewegt sich b zwischen 1.45 und 3.50 , bei einem Mittelwert von 2.33.

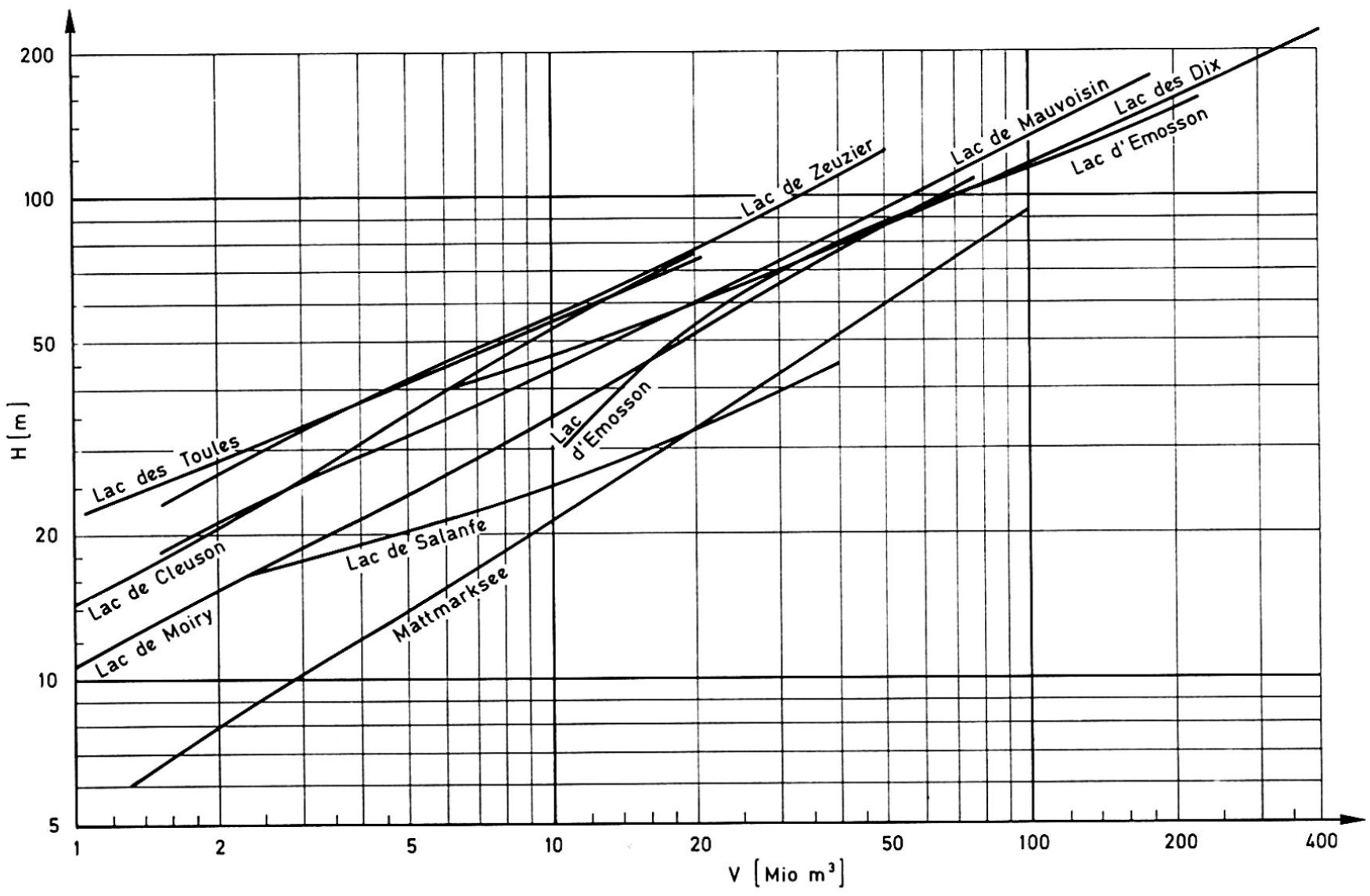

Bild 6: Speicherinhaltslinien, Flussgebiet Rhone 


\begin{tabular}{|l|r|ll|c|}
\hline Speicher & $\begin{array}{c}v_{\max } \\
\left(\text { Mio } \mathrm{m}^{3}\right)\end{array}$ & $\begin{array}{c}\mathrm{a} \\
\left(\mathrm{m}^{3-b}\right)\end{array}$ & $\begin{array}{c}\mathrm{b} \\
(-)\end{array}$ \\
\hline Lago di Vogorno & 106.0 & 2.00 & 3.36 \\
Lago di Luzzone & 88.0 & 2.94 & $10^{7}$ & 2.86 \\
Lago di Sambuco & 62.9 & 7.80 & $10^{3}$ & 1.94 \\
Lago Ritom & 53.9 & 1.54 & $10^{4}$ & 1.93 \\
Lago di Naret & 31.6 & 2.67 & $10^{2}$ & 2.52 \\
Lago di Cavagnoli & 28.9 & 2.38 & $10^{4}$ & 1.54 \\
Lago di Poschiavo & 111.1 & 1.72 & $10^{5}$ & 1.46 \\
Lago da l'Albigna & 70.0 & 5.62 & $10^{4}$ & 1.51 \\
Lago Bianco & 21.0 & 2.68 & $10^{1}$ & 3.40 \\
\hline
\end{tabular}

Tab. 5: Speicherkenngrössen, Flussgebiete Ticino, Adda

\section{Beckeninhalt natürlicher Seen}

In den vorangehenden Abschnitten ist gezeigt worden, dass für Stauseen die Abhängigkeit des Speicherinhaltes $\mathrm{V}$ von der Stauhöhe $\mathrm{H}$ durch das Potenzgesetz $V=a H^{b}$ angenähert werden kann. Ergänzend sei erwähnt, dass grundsätzlich der gleiche Ansatz auch bei natürlichen Seen für den Zusammenhang zwischen der Seetiefe $H$ und dem entsprechenden Beckeninhalt $V=V(H)$ gerechtfertigt ist. Anhand von Bild 2 kann man sich leicht idealisierte Beckenformen natürlicher Seen vorstellen, indem man sich den Längsschnitt symmetrisch zur eingezeichneten Talsperre ergänzt denkt. Die prinzipiellen Ueberlegungen im Abschnitt 2 über den Zusammenhang zwischen der Beckenform einerseits und den Beiwerten a und b der approximierenden Funktion $V=a H^{b}$ andererseits gelten analog auch für natürliche Seen.

Nachfolgend sind die Beiwerte festgehalten, welche für einige schweizerische Naturseen bestimmt wurden.

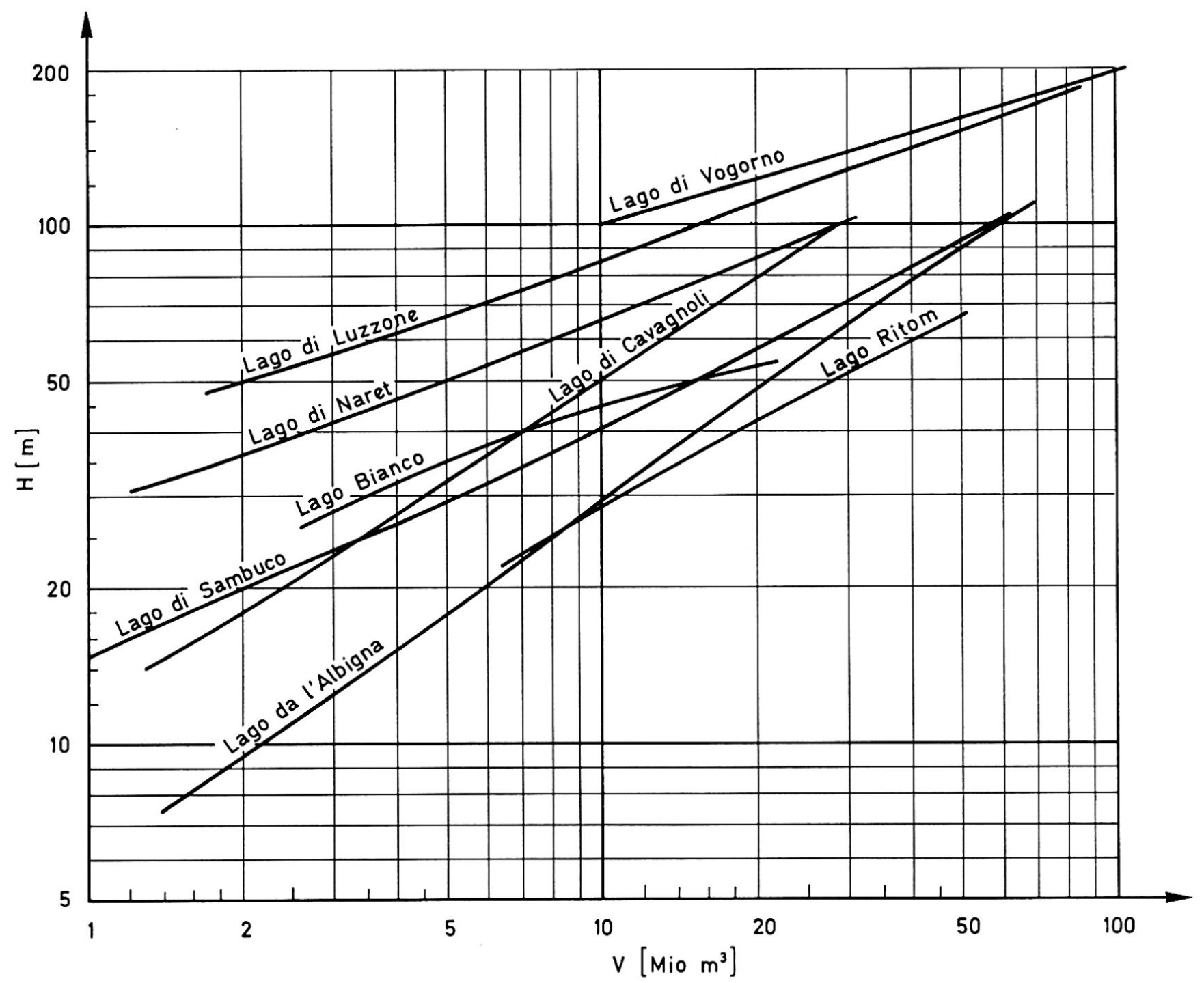

Bild 7: Speicherinhaltslinien, Flussgebiete Ticino, Adda 


\begin{tabular}{|l|l|l|}
\hline See & $a\left(\mathrm{~m}^{3-b}\right)$ & $b(-)$ \\
\hline Thunersee & $2.0410^{6}$ & 1.50 \\
Brienzersee & $1.9010^{6}$ & 1.42 \\
Walensee & $1.5510^{6}$ & 1.48 \\
Hallwilersee & $3.7410^{5}$ & 1.72 \\
Baldeggersee & $4.2110^{4}$ & 1.99 \\
Sempachersee & $2.2210^{5}$ & 1.94 \\
\hline
\end{tabular}

Tab. 6: Beiwerte $a$ und $b$ einiger Naturseen der Schweiz

Die Berechnung dieser Werte basiert auf Linien der Seefläche $F(H)$, welche aufgrund der Höhenkurven aus topographischen Karten (M 1:25000) planimetrisch ermittelt wurden. Die Beispiele in Tab. 6 deuten an, dass bei den schweizerischen Naturseen durchschnittlich kleinere Werte des Exponenten b, aber grössere a-Werte als bei den Speicherseen zu erwarten sind. Die kleineren b-Werte erklären sich daraus, dass - im Vergleich mit den Stauseen - viele natürliche Seen einen relativ flachen Seegrund aufweisen. Die grösseren a-Werte hängen mit den grösseren Seeoberflächen zusammen.

\section{Literatur}

(1) Eidg. Amt für Wasserwirtschaft: Die Seen der Schweiz. "Wasser, Energie, Luft", Heft 11/12, 1976.

(2) Sutherland, R.A.: Some Aspects of Water Conservation. Proceedings ASCE, Part I, Sept., 1930.

(3) Benk, D.: Ein Beitrag zum Betrieb und zur Bemessung von Hochwasserrückhal tebecken. Mitteilungen, Heft 17. Inst. f. Wasserwirtschaft, Grundbau und Wasserbau, Universität Stuttgart, 1971.

\section{Schlussfolgerungen}

Es zeigt sich, dass die Speicherinhaltslinien von Stauseen (und auch die Beckeninhaltslinien natürlicher Seen) im allgemeinen gut durch ein Potenzgesetz der Form $V(H)=a H^{b}$, mit zwei konstanten Beiwerten $a$ und $b$, angenähert werden können. Anhand der numerischen Werte von a und b lassen sich die Beckenformen der Stauseen untereinander vergleichen. Eine besondere Bedeutung kommt dabei dem Beiwert b zu. Kleine b-Werte charakterisieren Speicherseen, deren Spiegelfläche mit zunehmender Stauhöhe nur langsam anwächst. Typisch für solche Seen sind ein relativ flacher Seegrund und verhältnismässig steile Uferpartien. Grosse Beiwerte b kennzeichnen Becken, die sich nach oben sehr rasch erweitern. Entsprechende Stauseen weisen im Bereich der tiefsten Stelle oft einen schluchtartigen Grund auf. Dass für den Zusammenhang zwischen dem Speichervolumen $V$ und der Stauhöhe $\mathrm{H}$ ein Potenzgesetz von der Form $V=a H^{b}$ benutzt werden kann, ist vor allem aus wasserwirtschaftlicher sicht interessant: Verschiedene Probleme lassen sich mit $\mathrm{Hilfe}$ dieses Ansatzes rechnerisch merklich einfacher lösen. Selbst wenn die beiden Beiwerte a und b in gewissen Fällen nur näherungsweise bestimmt werden können, ermöglicht er eine sehr nützliche, einfache Abschätzung des Speicherinhaltes $V$ bei verschiedenen Stauhöhen $H$.

(4) Michels, V.S.: Reservoir Bas in Morphometry. Proceedings of the ASCE, Journal of the Irrigation and Drainage Division, March, 1977.

(5) Kühne, A.: Die rasche Absenkung von Stauseen. Optimale Programme für Stauseesysteme. Mitteilung $\mathrm{Nr} .23$ der Versuchsanstalt für Wasserbau, Hydrologie und Glaziologie an der ETH, Zürich, 1977.

(6) Eidg. Amt für Wasserwirtschaft: Statistik der Wasserkraftanlagen der Schweiz, Stand 1. Januar 1973. 